\title{
Germanium-on-silicon mid-infrared grating couplers with low-reflectivity inverse taper excitation
}

\author{
Carlos Alonso-Ramos ${ }^{1}$, Milos Nedeljkovic ${ }^{2, *}$, Daniel Benedikovic ${ }^{1}$, Jordi Soler \\ Penadés $^{2}$, Callum G. Littlejohns ${ }^{2,3}$, Diego Pérez-Galacho ${ }^{1}$, Laurent Vivien ${ }^{1}$, Pavel \\ Cheben $^{4}$, ANd Goran Z. MAshanovich ${ }^{2}$ \\ ${ }^{1}$ Centre for Nanoscience and Nanotechnology, CNRS, Univ. Paris-Sud, Universite Paris-Saclay, C2N - Orsay, 91405 Orsay cedex, France \\ ${ }^{2}$ Optoelectronics Research Centre, University of Southampton, Southampton SO17 1BJ, UK \\ ${ }^{3}$ Silicon Centre of Excellence, Nanyang Technological University, 50 Nanyang Avenue, Singapore, 639798, Singapore \\ ${ }^{4}$ National Research Council, Ottawa, K1A OR6, Canada \\ *Corresponding author: m.nedeljkovic@soton.ac.uk
}

Compiled August 19, 2016

\begin{abstract}
A broad transparency range of its constituent materials and compatibility with standard fabrication processes make germanium-on-silicon (Ge-on-Si) an excellent platform for the realization of mid-infrared photonic circuits. However, the comparatively large Ge waveguide thickness and its moderate refractive index contrast with the Si substrate hinder the implementation of efficient fiber-chip grating couplers. We report, for the first time, a single-etch Ge-on-Si grating coupler with an inversely tapered access stage, operating at $3.8 \mu \mathrm{m}$ wavelength. Optimized grating excitation yields a coupling efficiency of $-11 \mathrm{~dB}(7.9 \%)$, the highest value reported for a mid-infrared Ge-on-Si grating coupler, with reflectivity below $-15 \mathrm{~dB}(3.2 \%)$. The large periodicity of our higher-order grating design substantially relaxes fabrication constraints. We also demonstrate that a focusing geometry allows a ten-fold reduction in inverse taper length, from $500 \mu \mathrm{m}$ to $50 \mu \mathrm{m}$. ( 2016 Optical Society of America
\end{abstract}

OCIS codes: (130.3120) Integrated optics devices; (130.3060) Infrared; (050.1950) Diffraction gratings.

http://dx.doi.org/10.1364/ao.XX.XXXXXX

The demonstration of compact and efficient quantum cascade [1] and interband cascade [2] lasers with high output powers in the mid-infrared (MIR) wavelength range $(3 \mu \mathrm{m}-20 \mu \mathrm{m})$ has opened new opportunities for the development of integrated photonic devices with substantially extended wavelength range. High performance MIR photonic circuits are considered essential for many applications, including environmental monitoring [3], medical diagnostics [4], or free-space communications [5], to name a few. The potential impact of such applications has fueled the pursuit of suitable MIR photonic platforms in recent years [6]. In this context, group-IV materials such as silicon and germanium hold the unique potential to leverage the mature microelectronic facilities for cost-effective production of photonic devices at large volumes. A prohibitively large buried oxide absorption beyond $4 \mu \mathrm{m}$ restricts the implementation of silicon-on-insulator (SOI) platform in the MIR region [7]. Various solutions have been proposed to overcome this limitation, including partial [8] or complete [7,9] removal of the buried oxide, or the use of different substrates with wider transparency ranges, e.g. sapphire [10,11] and silicon nitride [12]. Alternatively, a promising approach is the use of a germanium guiding layer on a silicon substrate. The germanium-on-silicon (Ge-onSi) platform potentially offers many practical benefits for MIR photonic applications, including the wide transparency range of constituent materials as well as the compatibility with standard fabrication processes.

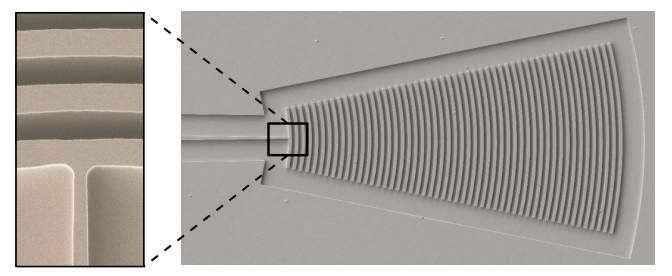

Fig. 1. Scanning electron microscope (SEM) image of the fabricated focusing grating coupler with an inverse taper excitation stage.

Since the first experimental demonstration of MIR Ge-on-Si waveguides in 2012 [13], several devices have been reported with steady progress in performance. These include low-loss waveguides and splitters [14], wavelength demultiplexers [1517], thermo-optical phase shifters [18] and all-optical modulators $[19,20]$. However, little attention has been dedicated to the development of suitable optical interfaces for Ge-on-Si technology, namely edge couplers and surface grating couplers. The difficulty of developing efficient Ge-on-Si edge couplers is two-fold. First, the current lack of MIR lensed fibers limits the performance of edge couplers [21]. Second, the preparation of high-quality facets in the Ge-on-Si platform is challenging [14]. Hence, the 
realization of grating-coupled optical ports that can directly interface cleaved optical fibers appears an optimal solution [14]. Grating couplers developed for the near-infrared applications have shown high coupling efficiencies [22? ] and low reflectivities [23, 24], with low Fabry-Pérot ripples [25]. The low reflectivity is particularly important for spectroscopic applications where absorption features with a bandwidth of a few hundreds $\mathrm{MHz}$ need to be precisely resolved [26].

Ge-on-Si waveguides entail significant challenges for the implementation of fiber-chip grating couplers. Specifically, the moderate index contrast between the Ge waveguide and the Si substrate $(\Delta n \sim 0.6)$ substantially limits the directionality and strength of the couplers. Furthermore, Ge-on-Si circuits use thick, typically $2-3 \mu \mathrm{m}$, Ge rib waveguides with low propagation loss [14]. Ge-on-Si waveguides have a comparatively large refractive index that results in strong back-reflections at the waveguide-to-grating interface. In addition, general guidelines to design first-order diffraction gratings cannot be directly transferred from the NIR to the MIR spectral range. The diffraction period of NIR grating couplers is typically chosen small enough to avoid higher diffraction orders [27]. However, this condition along with the deep etch required for small bending radii [17] can result in high aspect ratios for the MIR grating couplers (defined as the ratio between the etch depth and the width of the grating trench), that are difficult to fabricate.

In this letter we report, for the first time, the realization of single-etch step Ge-on-Si grating couplers with an inverse taper excitation, operating near $3.8 \mu \mathrm{m}$ wavelength. The inverse taper excitation stage advantageously maximizes the matching between the profiles of the grating mode and the excitation field, with a five-fold reflectivity reduction compared to the conventional taper. The grating is designed to operate in second diffraction order $(k=-2)$ under single-order radiation condition, substantially relaxing the aspect ratio requirements and facilitating device fabrication. We also demonstrate that a focusing geometry (see Fig. 1) allows ten-fold reduction in inverse taper length, specifically from $500 \mu \mathrm{m}$ to only $50 \mu \mathrm{m}$.
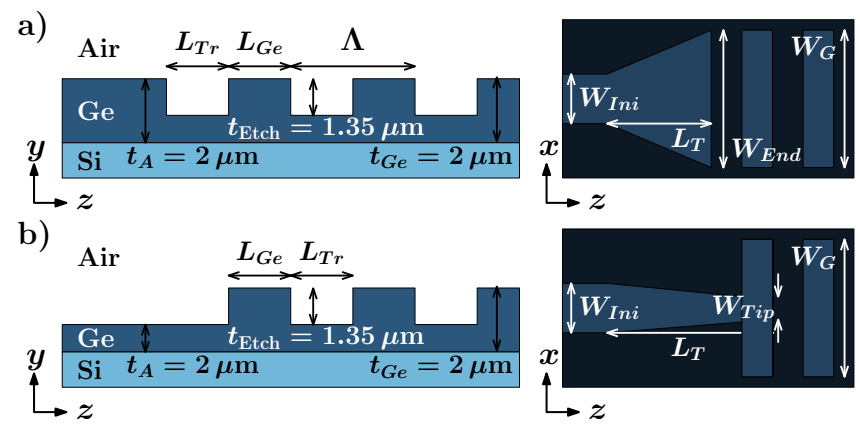

Fig. 2. Grating coupler schematic: excitation stage with (a) conventional taper and (b) inverse taper.

Figure 2 shows the grating coupler schematics. The grating period $\Lambda$, is defined as the sum of the trench $L_{T r}$ and tooth $L_{G e}$ lengths, with a duty cycle defined as $D C=L_{G e} / \Lambda$. For compatibility with previously developed functional blocks [17], we chose Ge waveguide thickness $t_{G e}=2 \mu \mathrm{m}$ and etch depth $t_{\mathrm{Ecth}}=1.35 \mu \mathrm{m}$. This way, the complete photonic circuit can be fabricated using a single-etch process. The grating coupler is interfaced by a cleaved fluoride MIR optical fiber that provides low propagation and bending loss. The optical fiber has a mode field diameter of $\sim 16 \mu \mathrm{m}$. A grating width of $26.1 \mu \mathrm{m}$, provides an overlap of $\sim 99 \%$ between the lateral ( $x$-axis) profile of the transverse electrical (TE) fundamental mode of the waveguide and the optical fiber mode. Following the approach proposed in [24], we analyze two vertical ( $y$ - $z$ plane) 2-D grating models, one comprising an access waveguide of thickness $t_{A}=2 \mu \mathrm{m}$ for a conventional taper excitation stage (see Fig.2(a)) and one with optimized access waveguide thickness of $t_{A}=t_{G e}-t_{\mathrm{Etch}}=$ $650 \mathrm{~nm}$, for an inverse taper design (see Fig.2(b)).

Grating coupler diffraction is governed by the phase matching condition:

$$
n_{u c} \sin \left(\theta_{k}\right)=n_{B}+\frac{k \lambda}{\Lambda}
$$

where $\lambda$ is the wavelength, $k$ is the diffraction order, $n_{B}$ is the effective index of the Bloch-Floquet mode in the grating region, $\theta_{k}$ is the radiation angle measured from the surface normal, and $n_{u c}$ is the refractive index of the upper cladding, here air $\left(n_{u c}=1\right)$. Diffraction orders that meet the radiation condition $\left|\sin \left(\theta_{k}\right)\right| \leqslant 1$ (i.e. yield a real value of $\theta_{k}$ ) are of primary importance for fiber-chip coupling [27]. Multiple diffraction orders propagating at different angles would result in poor coupling efficiency. Taking into account that the effective index of the Bloch-Floquet mode is larger than the refractive index of the superstrate $\left(n_{B}>n_{u c}=1\right)$, it can be shown that only negative diffraction orders can be radiated out of the coupling structure. Hence, conventional grating designs yield single-beam radiation by choosing a period small enough to ensure that only the first diffraction order $(k=-1)$ meets the radiation condition. It can be derived from Eq. (1) that single-beam radiation condition can be achieved for any (negative) diffraction order $k$ given that $n_{B}>-k n_{u c}$. This way, Ge-on-Si gratings can benefit from its high effective index to yield single-beam radiation for higher diffraction orders. Such designs exhibit comparatively larger periods that relax aforementioned fabrication constraints. To illustrate this behavior, in Fig. 3(a) we show the radiation angle $\theta_{k}$, calculated from Eq. (1), as a function of the grating period for different diffraction orders $(k=-1 ; k=-2$ and $k=-3)$. Here, we set a duty cycle of $D C=0.5$ and approximate the Bloch-Floquet mode index by the average of the indices in the etched and un-etched grating regions [28], obtaining a value of $n_{B} \sim 3.8$. Three regions can be distinguished (shadowed regions in Fig. 3(a)). Within these regions, only one order meets the radiation condition (yields a radiation angle with a real value), thus ensuring single-beam radiation. The first window, with the shortest period (around $1 \mu \mathrm{m}$ ), corresponds to the conventional first-order $(k=-1)$ diffraction regime, whilst the other two correspond to higher order $(k=-2$ and $k=-3)$ operation. This way, a grating coupler with a radiation angle of $\theta \sim 15^{\circ}$ would require $L_{T r}=550 \mathrm{~nm}$ and $t_{\mathrm{Etch}}=1.35 \mu \mathrm{m}$ for $k=-1$, compared to $L_{T r}=1.1 \mu \mathrm{m}$ and $t_{\text {Etch }}=1.35 \mu \mathrm{m}$ for $k=-2$. Indeed, our $2^{\text {nd }}$ order design significantly relaxes the aspect ratio requirements and eases the manufacturing, releasing new degrees of freedom in device optimization.

The coupler performance in terms of the directionality, the reflectivity and the overall coupling efficiency are analyzed by using a 2-D Fourier expansion simulator [29]. Here, the fundamental TE mode of the access waveguide is used as an excitation stage. Figure 3(b) shows calculated reflectivity as a function of the grating period for conventional and inverse taper excitation stages. When the conventional taper is used for grating excitation (blue line in Fig. 3(b)), we predict a reflectivity exceeding $25 \%$, even for radiation angles far from the Bragg condition. This high reflectivity arises from the mismatch between the fundamental mode of the $2 \mu \mathrm{m}$ thick access waveguide and that of 
a)

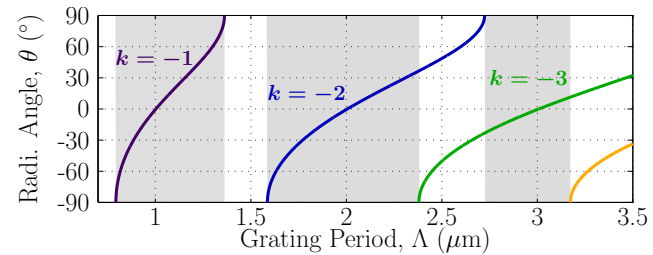

b)

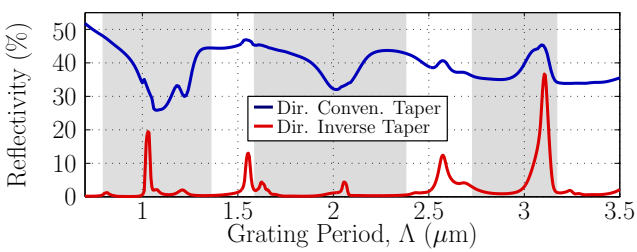

c)

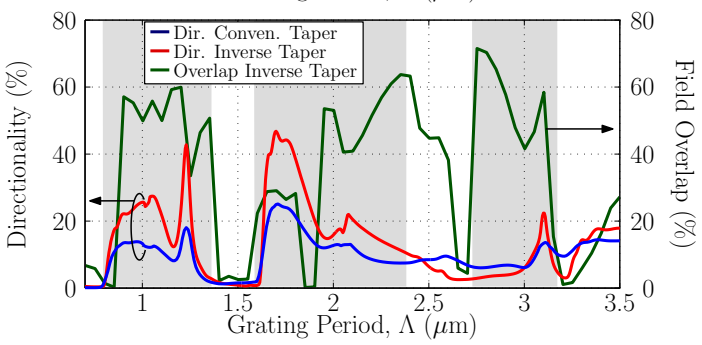

Fig. 3. (a) Radiation angle in Eq. (1) as a function of the grating period for different diffraction orders, considering $n_{B} \sim$ 3.8. Calculated (b) back-reflections and (c) directionality and field overlap with the fundamental mode of the fiber as a function of the grating period, for a coupler with $D C=0.5$.

the grating region. When the inverse coupler excitation stage is used (i.e. the same thickness for access waveguide as for the etched grating region) matching with the grating mode is maximized [24] and back-reflections are reduced to less than $0.5 \%$ (red line in Fig. 3(b)). An additional advantage of this approach is that both the grating region and the interconnecting waveguides can be defined in the same fabrication step, easing the fabrication process.

In Fig. 3(c), we compare grating directionality $(\Gamma)$, defined as the ratio between power radiated upwards and the total radiated power (in upwards and downwards directions), as a function of the grating period for the conventional and inverse taper excitations. The inverse taper design exhibits a substantial directionality improvement, e.g. from $\Gamma \sim 30 \%$ to $\Gamma \sim 60 \%$ for $\Lambda=1.75 \mu \mathrm{m}$. In Fig. 3(c) we plot calculated field overlap with the fundamental mode of the fiber as a function of the grating period. Due to a reduced radiation strength, the high directionality design $(\Lambda=1.75 \mu \mathrm{m})$ yields a field overlap of only $25.5 \%$, resulting in a coupling efficiency of $15 \%$. Conversely, the coupler design with a period of $\Lambda=2.2 \mu \mathrm{m}$ exhibits a moderate directionality $(\Gamma \sim 20 \%$ ) with improved field overlap of $51 \%$, resulting in a coupling efficiency to the fiber of $11.5 \%$. Although being out of the scope of this work, which targets the first demonstration the inverse-taper-based Ge-on-Si coupler, it is worth mentioning that the directionality can be further improved with a bottom mirror to harvest the power radiated to the substrate [22].

The design of the inverse taper that connects the single-mode rib waveguide (of $W_{\text {Ini }}=2.1 \mu \mathrm{m}$ width) to the grating region includes the optimization of the taper tip $\left(W_{\text {Tip }}\right)$ and the length $\left(L_{T}\right)$, schematically shown in Fig. 2(b). We define the optimum excitation field as that having a distribution along the $y$-axis that minimizes the reflectivity, and a profile along the $x$-axis that maximizes overlap with the Gaussian-like mode of the optical fiber [30]. In Fig. 4, we show calculated field overlap between the mode at the taper tip and the optimum excitation field. We chose a value of $W_{\text {Tip }}=450 \mathrm{~nm}$, compatible with the minimum feature size of our fabrication process, that yields large field overlap of $95 \%$. As shown in the inset of Fig. 4, rib width reduction confines the field in the lower slab and expands it in the lateral direction. As a result of this vertical and lateral field distribution transformation, the inverse taper requires a length of $>500 \mu \mathrm{m}$ the and insertion loss of $\leqslant 0.2 \mathrm{~dB}$. However, this comparatively long taper implies an increased interaction of the field with side-wall roughness of the rib section and the dislocations close to the Ge-to-Si boundary, resulting in potentially large taper propagation loss. This issue can be alleviated by reducing the taper length using a focusing grating geometry [31, 32]. In this case, grating lines are arranged along confocal ellipses with a focal point at the end of the interconnection waveguide, thereby enabling taper lengths of only a few tens of micrometers.

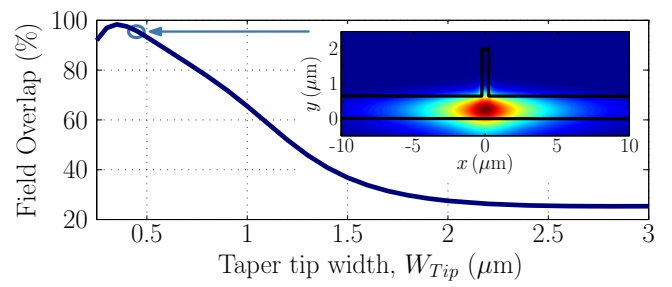

Fig. 4. Field overlap between optimum excitation field and fundamental mode at the taper tip as a function of the tip width $\left(W_{\text {Tip }}\right)$. Inset: profile of fundamental TE-polarized mode for $W_{\text {Tip }}=450 \mathrm{~nm}$.

To experimentally evaluate these different grating geometries, we have fabricated nominally identical grating couplers with three different excitation stages, namely conventional, inverse and inverse-focusing tapers. Note that the high directionality design $(\Lambda=1.75 \mu \mathrm{m})$ exhibits an impractical radiation angle of $\theta_{-2}=-41^{\circ}$. Hence, we chose a moderate directionality design with $\Lambda=2.2 \mu \mathrm{m}$ that yields a radiation angle of $\theta_{-2}=$ $15^{\circ}$ with comparable coupling efficiency. Conventional tapers have an end-width of $W_{E n d}=26.1 \mu \mathrm{m}$ and a length of $L_{T}=$ $500 \mu \mathrm{m}$. Inverse tapers have an tip width of $W_{\text {Tip }}=450 \mathrm{~nm}$ and a length of $L_{T}=500 \mu \mathrm{m}$. The focusing geometry (with initial and final grating widths of $20 \mu \mathrm{m}$ and $40 \mu \mathrm{m}$ respectively) substantially reduces the inverse taper length to $L_{T}=50 \mu \mathrm{m}$, for the same tip width of $W_{\text {Tip }}=450 \mathrm{~nm}$. The devices were fabricated on Ge-on-Si wafer with a $2 \mu \mathrm{m}$ thick Ge layer. Electronbeam lithography was used to pattern a ZEP-520A positive resist, followed by ICP dry etching (with $\mathrm{SF}_{6}$ and $\mathrm{C}_{4} \mathrm{~F}_{8}$ gases) to define the structures. Figure 1 shows scanning electron microscope images of the fabricated inverse taper with the focusing grating.

To characterize the fiber-chip coupling efficiency, input and output grating couplers were connected in a back-to-back configuration. Measured coupling efficiency is shown in Fig. 5 for the three excitation stages. From the $20 \mathrm{~nm}$ red-shift in the peak efficiency, we estimated a $100 \mathrm{~nm}$ shallower etching of the grating teeth. This produced a reflectivity reduction for the conventional taper and a detrimental sub-optimal inverse taper excitation. Still, the couplers with inverse taper and inversefocusing geometry exhibit a five-fold reflectivity improvement compared to the conventional taper ( $\sim 1 \mathrm{~dB}$ Fabry-Pérot ripple), yielding a reflectivity below $-15 \mathrm{~dB}(<0.2 \mathrm{~dB}$ Fabry-Pérot ripple). Nevertheless, the narrow width and comparatively large 


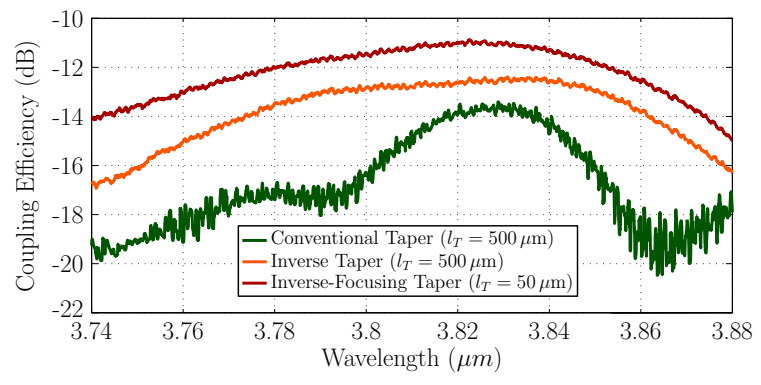

Fig. 5. Measured coupling efficiency as a function of the wavelength for nominally identical grating couplers with conventional, inverse taper and inverse-focusing excitation stages.

length of the inverse taper makes it more sensitive to small width variations that affect the distribution of the grating excitation field. This, together with the longer interaction with side-wall roughness and defects in the Ge-to-Si boundary compromise the efficiency of the inverse tapered coupler. Our $50 \mu \mathrm{m}$ long inverse-focusing grating coupler overcomes these limitations providing $\mathrm{a} \sim 1.5 \mathrm{~dB}$ improvement compared to the inverse taper coupler and $\mathrm{a} \sim 2.5 \mathrm{~dB}$ improvement compared to the conventional taper to yield a coupling efficiency of $-11 \mathrm{~dB}(7.9 \%)$, with a $1 \mathrm{~dB}$ bandwidth of $70 \mathrm{~nm}$.

In conclusion, we reported the design and experimental characterization of surface grating couplers implemented in $2 \mu \mathrm{m}$ thick Ge-on-Si waveguides for the MIR wavelength range near $3.8 \mu \mathrm{m}$. Our calculations showed that inverse taper excitation can substantially improve grating directionality from $30 \%$ to $60 \%$. By exploiting the inverse taper concept, we substantially reduced the measured grating reflectivity $(<-15 \mathrm{~dB})$ while at the same time using single-etch fabrication of the interconnecting waveguides and the coupler. We demonstrated, for the first time, the advantageous combination of short (50 $\mu \mathrm{m}$ long) inverse taper and focusing grating geometry, yielding a fiber-chip coupling efficiency of $-11 \mathrm{~dB}(7.9 \%)$, which is to the best of our knowledge, the highest coupling efficiency yet experimentally demonstrated for a Ge-on-Si grating coupler in the MIR. The coupling efficiency could be further increased by using a bottom mirror [22]. We also provided comprehensive guidelines for the design of higher-order gratings with relaxed fabrication constraints. This work represents an important step towards the realization of high-performance surface grating couplers for the MIR Ge-on-Si photonic platform.

\section{FUNDING INFORMATION}

The authors acknowledge the Engineering and Physical Sciences Research Council (EPSRC) for financial support under the project EP/ L01162X/1 (MIGRATION), G. Z. Mashanovich acknowledges support from the Royal Society through his University Research Fellowship and C. G. Littlejohns' work was supported by National Research Foundation of Singapore (NRFCRP12- 2013-04). The data for this work is accessible through the University of Southampton Institutional Research Repository (DOI: $x x x$ ).

\section{REFERENCES}

1. M. Razeghi, Q. Y. Lu, N. Bandyopadhyay, W. Zhou, D. Heydari, Y. Bai, and S. Slivken, Opt. Express 23, 8462 (2015)

2. C. Borgentun, C. Frez, R. M. Briggs, M. Fradet, and S. Forouhar, Opt. Express 23, 2446 (2015).
3. T. Schädle, B. Pejcic, M. Myers, and B. Mizaikoff, Anal. Chem. 86, 9512 (2014).

4. K.-Z. Liu, M. Xu, and D. A. Scott, Clin. Pharmacol. Ther. 69, 89 (2001).

5. E. Leitgeb, T. Plank, M. S. Awan, P. Brandl, W. Popoola, Z. Ghassemloy, F. Ozek, and M. Witting, in IEEE Int. Conf. on Transparent Optical Networks (2010).

6. J. Hu, J. Meyer, K. Richardson, and L. Shah, Opt. Mater. Express 3, 1571 (2013)

7. J. Soler Penadés, C. Alonso-Ramos, A. Z. Khokhar, M. Nedeljkovic, L. A. Boodhoo, A. Ortega-Moñux, I. Molina-Fernández, P. Cheben, and G. Z. Mashanovich, Opt. Lett. 39, 5661 (2014).

8. P. T. Lin, V. Singh, J. Wang, H. Lin, J. Hu, K. Richardson, J. D. Musgraves, I. Luzinov, J. Hensley, L. C. Kimerling, and A. Agarwal, Opt. Mat. Express 3, 1474 (2013).

9. X. Wang, Z. Cheng, K. Xu, H. K. Tsang, and J.-B. Xu, Nature Photon. 7, 888 (2013).

10. T. Baehr-Jones, A. Spott, R. llic, A. Spott, B. Penkov, W. Asher, and M. Hochberg, Opt. Express 18, 12127 (2010).

11. N. Singh, D. D. Hudson, Y. Yu, Ch. Grillet, S. D. Jackson, A. CasasBedoya, A. Read, P. Atanackovic, S. G. Duvall, S. Palomba, B. LutherDavies, S. Madden, D. J. Moss, and B. J. Eggleton, Optica 2, 797 (2015).

12. J. Mu, R. Soref, L. C. Kimerling, and J. Michel, Appl. Phys. Lett. 104, 031115 (2014).

13. Y.-C. Chang, V. Paeder, L. Hvozdara, J.-M. Hartmann, and H. P. Herzig, Opt. Lett. 37, 2883 (2012).

14. M. Nedeljkovic, J. Soler Penadés, C. J. Mitchell, A. Z. Khokhar, S. Stanković, T. Dominguez Bucio, C. G. Littlejohns, F. Y. Gardes, and G. Z. Mashanovich, IEEE Photon. Technol. Lett. 27, 1040 (2015).

15. A. Malik, M. Muneeb, S. Pathak, Y. Shimura, J. Van Campenhout, R. Loo, and G. Roelkens, IEEE Photon. Technol. Lett. 25, 1805 (2013).

16. A. Malik, M. Muneeb, Y. Shimura, J. Van Campenhout, R. Loo, and G. Roelkens, Appl. Phys. Lett. 103, 191119 (2013).

17. B. Troia, J. Soler Penadés, A. Z. Khokhar, M. Nedeljkovic, C. AlonsoRamos, V. M. N. Passaro, and G. Z. Mashanovich, Opt. Lett. 41, 610 (2016).

18. A. Malik, S. Dwivedi, L. Van Landschoot, M. Muneeb, Y. Shimura, G. Lepage, J. Van Campenhout, W. Vanherle, T. Van Opstal, R. Loo, and G. Roelkens, Opt. Express 22, 28479 (2014).

19. L. Shen, N. Healy, C. J. Mitchell, J. Soler Penadés, M. Nedeljkovic, G. Z. Mashanovich, and A. C. Peacock, Opt. Lett. 40, 268 (2015).

20. L. Shen, N. Healy, C. J. Mitchell, J. Soler Penadés, M. Nedeljkovic, G. Z. Mashanovich, and A. C. Peacock, Opt. Lett. 40, 2213-2216 (2015).

21. C. J. Smith, R. Shankar, M. Laderer, M. B. Frish, M. Lončar, and M. G. Allen, Opt. Express 23, 5491 (2015).

22. D. Benedikovic, P. Cheben, J. H. Schmid, D.-X. Xu, B. Lamontagne,S. Wang, J. Lapointe, R. Halir, A. Ortega-Moñux, S. Janz, and M. Dado, Opt. Express 23, 22628 (2015).

23. Y. Li, L. Li, B. Tian, G. Roelkens, and R. Baets, IEEE Photon. Technol. Lett. 25, 1195 (2013).

24. C. Alonso-Ramos, A. Ortega-Moñux, L. Zavargo-Peche, R. Halir, J. de Oliva-Rubio, I. Molina-Fernández, P. Cheben, D.-X. Xu, S. Janz, N. Kim, and B. Lamontagne, Opt. Lett. 36, 2647 (2011).

25. W. Zhang, S. Serna, X. Le Roux, C. Alonso-Ramos, L. Vivien, and E. Cassan, Opt. Lett. 40, 5566 (2015).

26. G. Millot, S. Pitois, M. Yan, T. Hovhannisyan, A. Bendahmane, T. W. Hänsch, and N. Picqué, Nature Photon. 10, 27 (2016).

27. T. Tamir, and S. Peng, Appl. Phys. A-Mater. 14, 235 (1977).

28. S. M. Rytov, Sov. Phys. JETP 2, 466 (1956).

29. L. Zavargo-Peche, A. Ortega-Moñux, J. G. Wangüemert-Pérez, and I. Molina-Fernández, Prog. Electromagn. Res. 123, 447 (2012).

30. C. Alonso-Ramos, A. Ortega-Moñux, I. Molina-Fernández, P. Cheben, L. Zavargo-Peche, and R. Halir, Opt. Express 18, 15189 (2010).

31. R. Waldhäusl, B. Schnabel, P. Dannberg, E.-B. Kley, A. Bräure, and W. Karthe, Appl. Opt. 36, 9383 (1997).

32. F. Van Laere, T. Claes, J. Schrauwen, S. Scheerlinck, W. Bogaerts, D. Taillaert, Liam O'Faolain, D. Van Thourhout, and R. Baets, IEEE Photon. Technol. Lett. 23, 1919 (2007). 


\section{REFERENCES}

1. M. Razeghi, Q. Y. Lu, N. Bandyopadhyay, W. Zhou, D. Heydari, Y. Bai, and S. Slivken, "Quantum cascade lasers: from tool to product," Opt. Express 23, 8462-8475 (2015).

2. C. Borgentun, C. Frez, R. M. Briggs, M. Fradet, and S. Forouhar, "Single-mode high-power interband cascade lasers for mid-infrared absorption spectroscopy," Opt. Express 23, 2446-2450 (2015).

3. T. Schädle, B. Pejcic, M. Myers, and B. Mizaikoff, "Fingerprinting oils in water via their dissolved VOC pattern using mid-infrared sensors," Anal. Chem. 86, 9512-9517 (2014).

4. K.-Z. Liu, M. Xu, and D. A. Scott, "Biomolecular characterisation of leucocytes by infrared spectroscopy," Clin Pharmacol. Ther. 69, 89-95 (2001).

5. E. Leitgeb, T. Plank, M. S. Awan, P. Brandl, W. Popoola, Z. Ghassemloy, F. Ozek, and M. Witting, "Analysis and evaluation of optimum wavelengths for free-space optical transceivers," in IEEE Int. Conf. on Transparent Optical Networks (2010) pp.1-7.

6. J. Hu, J. Meyer, K. Richardson, and L. Shah, "Feature issue introduction: mid-IR photonic materials," Opt. Mater. Express 3, 1571-1575 (2013).

7. J. Soler Penadés, C. Alonso-Ramos, A. Z. Khokhar, M. Nedeljkovic, L. A. Boodhoo, A. Ortega-Moñux, I. Molina-Fernández, P. Cheben, and G. Z. Mashanovich, "Suspended SOI waveguide with sub-wavelength grating cladding for mid-infrared," Opt. Lett. 39, 5661-5664 (2014).

8. P. T. Lin, V. Singh, J. Wang, H. Lin, J. Hu, K. Richardson, J. D. Musgraves, I. Luzinov, J. Hensley, L. C. Kimerling, and A. Agarwal, "SiCMOS compatible materials and devices for mid-IR microphotonics," Opt. Mat. Express 3, 1474-1487 (2013).

9. X. Wang, Z. Cheng, K. Xu, H. K. Tsang, and J.-B. Xu, "High-responsivity graphene/silicon-heterostructure waveguide photodetectors," Nature Photon. 7, 888-891 (2013).

10. T. Baehr-Jones, A. Spott, R. Ilic, A. Spott, B. Penkov, W. Asher, and M. Hochberg, "Silicon-on-sapphire integrated waveguides for the midinfrared," Opt. Express 18, 12127-12135 (2010).

11. N. Singh, D. D. Hudson, Y. Yu, Ch. Grillet, S. D. Jackson, A. CasasBedoya, A. Read, P. Atanackovic, S. G. Duvall, S. Palomba, B. LutherDavies, S. Madden, D. J. Moss, and B. J. Eggleton, "Mid-infrared supercontinuum generation from 2 to $6 \mu \mathrm{m}$ in a silicon nanowire," Optica 2, 797-802 (2015).

12. J. Mu, R. Soref, L. C. Kimerling, and J. Michel, "Silicon-on-nitride structures for mid-infrared gap-plasmon waveguiding," Appl. Phys. Lett. 104, 031115 (2014).

13. Y.-C. Chang, V. Paeder, L. Hvozdara, J.-M. Hartmann, and H. P. Herzig, "Low-loss germanium strip waveguides on silicon for the mid-infrared," Opt. Lett. 37, 2883-2885 (2012).

14. M. Nedeljkovic, J. Soler Penadés, C. J. Mitchell, A. Z. Khokhar, S. Stanković, T. Dominguez Bucio, C. G. Littlejohns, F. Y. Gardes, and G. Z. Mashanovich, "Surface-grating-coupled low-loss Ge-on-Si rib waveguides and multimode interferometers," IEEE Photon. Technol. Lett. 27, 1040-1043 (2015).

15. A. Malik, M. Muneeb, S. Pathak, Y. Shimura, J. Van Campenhout, R. Loo, and G. Roelkens, "Germanium-on-silicon mid-infrared arrayed waveguide grating multiplexers," IEEE Photon. Technol. Lett. 25, 18051808, (2013).

16. A. Malik, M. Muneeb, Y. Shimura, J. Van Campenhout, R. Loo, and G. Roelkens, "Germanium-on-silicon planar concave grating wavelength (de)multiplexers in the mid-infrared," Appl. Phys. Lett. 103, 191119161119-4, (2013).

17. B. Troia, J. Soler Penadés, A. Z. Khokhar, M. Nedeljkovic, C. AlonsoRamos, V. M. N. Passaro, and G. Z. Mashanovich, "Germanium-onsilicon Vernier-effect photonic microcavities for the mid-infrared," Opt. Lett. 41, 610-613 (2016).

18. A. Malik, S. Dwivedi, L. Van Landschoot, M. Muneeb, Y. Shimura, G. Lepage, J. Van Campenhout, W. Vanherle, T. Van Opstal, R. Loo, and G. Roelkens, "Ge-on-Si and Ge-on-SOI thermo-optic phase shifters for the mid-infrared," Opt. Express 22, 28479-28488 (2014).

19. L. Shen, N. Healy, C. J. Mitchell, J. Soler Penadés, M. Nedeljkovic, G. Z. Mashanovich, and A. C. Peacock, "Mid-infrared all-optical modulation in germanium-on-silicon waveguides," Opt. Lett. 40, 268-271 (2015).
20. L. Shen, N. Healy, C. J. Mitchell, J. Soler Penadés, M. Nedeljkovic, G. Z. Mashanovich, and A. C. Peacock, "Two-photon absorption and all-optical modulation in germanium-on-silicon waveguides for the midinfrared," Opt. Lett. 40, 2213-2216 (2015).

21. C. J. Smith, R. Shankar, M. Laderer, M. B. Frish, M. Lončar, and M. G. Allen, "Sensing nitrous oxide with QCL-coupled silicon-on-sapphire ring resonators," Opt. Express 23, 5491-5499 (2015).

22. D. Benedikovic, P. Cheben, J. H. Schmid, D.-X. Xu, B. Lamontagne,S. Wang, J. Lapointe, R. Halir, A. Ortega-Moñux, S. Janz, and M. Dado, "Subwavelength index engineered surface grating coupler with subdecibel efficiency for 220-nm silicon-on-insulator waveguides," Opt. Express 23, 22628-22635 (2015)

23. Y. Li, L. Li, B. Tian, G. Roelkens, and R. Baets, "Reflectionless tilted grating couplers with improved coupling efficiency based on a silicon overlay," IEEE Photon. Technol. Lett. 25, 1195-1198 (2013).

24. C. Alonso-Ramos, A. Ortega-Moñux, L. Zavargo-Peche, R. Halir, J. de Oliva-Rubio, I. Molina-Fernández, P. Cheben, D.-X. Xu, S. Janz, N. $\mathrm{Kim}$, and B. Lamontagne, "Single-etch grating coupler for micrometric silicon rib waveguides," Opt. Lett. 36, 2647-2649 (2011).

25. W. Zhang, S. Serna, X. Le Roux, C. Alonso-Ramos, L. Vivien, and E. Cassan, "Analysis of silicon-on-insulator slot waveguide ring resonators targeting high Q-factors," Opt. Lett. 40, 5566-5569 (2015).

26. G. Millot, S. Pitois, M. Yan, T. Hovhannisyan, A. Bendahmane, T. W. Hänsch, and N. Picqué, "Frequency-agile dual-comb spectroscopy," Nature Photon. 10, 27-30 (2016).

27. T. Tamir, and S. Peng, "Analysis and design of grating couplers," Appl. Phys. A-Mater. 14, 235-254 (1977).

28. S. M. Rytov, "The electromagnetic properties of finely layered medium," Sov. Phys. JETP 2, 466-475, (1956).

29. L. Zavargo-Peche, A. Ortega-Moñux, J. G. Wangüemert-Pérez, and I. Molina-Fernández, "Fourier based combined techniques to design novel sub-wavelength optical integrated devices," Prog. Electromagn. Res. 123, 447-465, (2012).

30. C. Alonso-Ramos, A. Ortega-Moñux, I. Molina-Fernández, P. Cheben, L. Zavargo-Peche, and R. Halir, "Efficient fiber-to-chip grating coupler for micrometric SOI rib waveguides," Opt. Express 18, 15189-15200 (2010).

31. R. Waldhäusl, B. Schnabel, P. Dannberg, E.-B. Kley, A. Bräure, and W. Karthe, "Efficient coupling into polymer waveguides by gratings," Appl. Opt. 36, 9383-9390 (1997).

32. F. Van Laere, T. Claes, J. Schrauwen, S. Scheerlinck, W. Bogaerts, D. Taillaert, Liam O'Faolain, D. Van Thourhout, and R. Baets, "Compact focusing grating couplers for silicon-on-insulator integrated circuits," IEEE Photon. Technol. Lett. 23, 1919-1921 (2007). 